\title{
Neural Responses in the Retinotopic Representation of the Blind Spot in the Macaque V1 to Stimuli for Perceptual Filling-In
}

\author{
Hidehiko Komatsu, Masaharu Kinoshita, and Ikuya Murakami \\ Laboratory of Neural Control, National Institute for Physiological Sciences, Myodaiji, Okazaki-shi, Aichi, 444-8585 Japan
}

\begin{abstract}
When visual stimuli that cover the entire blind spot are presented monocularly, the color and brightness of the surrounding field are seen within the blind spot, although it receives no retinal input. Important questions about such perceptual filling-in are whether neurons in the visual system representing visual field locations within the blind spot are activated when filling-in occurs and, if so, what the properties of these neurons are. To address these questions, we recorded the activities of single neurons in the primary visual cortex (V1) of the awake monkey. We first identified the area of $\mathrm{V} 1$ representing the region of the blind spot and then assessed neural responses to stationary visual stimuli of various size. We found that there are neurons in layer 4 and
\end{abstract}

deeper laminae, particularly layer 6 , that respond to large stimuli covering the blind spot which induces perceptual filling-in. Most of these neurons had very large binocular receptive fields that extended outside the blind spot. These neurons also preferred relatively large stimuli and exhibited color selectivity. These results indicate that when a large uniform surface is presented on the blind spot, neurons at the $\mathrm{V} 1$ region representing the blind spot transmit signals essential for filling-in that inform of the presence of a large surface as well as the absence of smaller stimuli at the blind spot.

Key words: filling-in; blind spot; V1; monkey; surface perception; completion; visual perception
When we look at a surface of homogeneous color and luminance, light rays coming into the eye from each point within the surface area stimulate retinal photoreceptors, and this point-wise information may be the basis of our perception of color and luminance. At the blind spot, however, there is no retinal input; thus no such point-wise signal is available. Despite this, we perceive some color and brightness within the blind spot as if the stimulus attributes in the surround had filled it in (Ramachandran, 1992). This phenomenon is called perceptual filling-in (Walls, 1954; Gerrits and Vendrik, 1970; Ramachandran and Gregory, 1991; see Pessoa et al., 1998) and is not unique to humans (Komatsu and Murakami, 1994a).

Retinal topography is preserved in the primary visual cortex (V1) and in many extrastriate visual areas (Daniel and Whitteridge, 1961; Van Essen and Zeki, 1978; Gattass et al., 1981, 1988). When we look at a surface, a region of the visual cortex that corresponds topographically to the surface is stimulated, and some of the neurons there are activated. Such topographic correspondence is thought to be a neural basis of surface perception. Several recent studies provide cumulative evidence that both the contour and interior of a surface are represented by the activities of V1 neurons (Lamme, 1995; Komatsu et al., 1996; Rossi et al., 1996; Zipser et al., 1996). However, perceptual filling-in at the blind spot raises an important question about the relationship between topographic activities and surface perception. When a homogeneous surface covers the blind spot, the corresponding region of the retinotopic map of the cortex would not receive afferent inputs as other regions do; nevertheless, we see a homogeneous surface, not a surface with a blind spot-shaped hole. Where in the brain is the perceived interior of the blind spot represented? If surface perception requires V1 neurons to encode the interior of a surface, V1

Received Aug. 16, 2000; revised Oct. 2, 2000; accepted Oct. 3, 2000.

This work is supported by the "Research for the Future" Program from The Japan Society for the Promotion of Science Grant 96L00202 and by the Japanese Ministry of Education Grant 08279102 . We are grateful to Dr. Minami Ito for comments on a previous version of this manuscript. We thank M. Okui, M. Togawa, and M. Yoshitomo for technical assistance.

Correspondence should be addressed to Dr. Hidehiko Komatsu at the above address. E-mail: komatsu@nips.ac.jp.

Dr. Murakami's present address: Department of Human and Information Science Lab, NTT Communication Science Labs, 3-1 Morinosato Wakamiya, Atsugi, Kanagawa, 243-0198 Japan.

Copyright (C) 2000 Society for Neuroscience $0270-6474 / 00 / 209310-10 \$ 15.00 / 0$ neurons in the retinotopic representation of the blind spot (hereafter referred to as "BS representation") should be activated when filling-in occurs at the blind spot.

Fiorani et al. (1992) reported previously that in anesthetized monkeys, a bar moving across the blind spot generates a neural response in the retinotopic representation of the blind spot in V1. To date, however, there have been no attempts to study neural responses related to filling-in of a surface at the blind spot or to any type of filling-in there in an awake preparation. We therefore recorded neuron activities from the BS representation in V1 of the awake monkey and analyzed their visual responses. We addressed two main questions. Are neurons in the BS representation in V1 activated when filling-in occurs? And if so, what are the properties of these neurons?

Parts of this paper have been reported previously (Komatsu and Murakami, 1994b; Komatsu et al., 1995).

\section{MATERIALS AND METHODS}

Behavioral task. Three macaque monkeys (Macaca fuscata) were used for the experiments. During the experiments, the monkeys sat in a primate chair and faced a computer display [either a Mitsubishi XC3715C (Tokyo, Japan; $640 \times 400$ pixels; subtending $21 \times 13^{\circ} ; 173 \mathrm{~cm}$ from the monkeys' eyes; 60 frames $/ \mathrm{sec}$ ) or a Nanao Flex Scan 54T (Tokyo, Japan; $1024 \times 768$ pixels; subtending $34 \times 26^{\circ} ; 57 \mathrm{~cm}$ from the monkeys' eyes; 60 frames $\left.\left./ \mathrm{sec}\right)\right]$.

Each monkey was first trained to perform a fixation task (Wurtz, 1969). A trial started when a small stationary spot (fixation spot) appeared on the screen. The monkey was required to look at the fixation spot within 400 msec and then to maintain fixation within an electronic window $\left(1 \times 1^{\circ}\right)$ around the fixation spot for the remainder of the trial. During fixation, another visual stimulus, which was used to study the visual responses of neurons, was presented for $400 \mathrm{msec}$. The task was controlled by a personal computer (NEC PC9801, Tokyo, Japan), and visual stimuli were generated using a graphics board (Digital Arts Hyper-Frame, Tokyo, Japan, or FORCE GP-1122N, Tokyo, Japan) in the same computer. Eye position was monitored using the magnetic search-coil technique (Robinson, 1963). If the monkey kept its gaze within the electronic window until the end of the trial, it was given a drop of water as a reward; otherwise the trial was terminated without reward. Trials were separated by $3 \mathrm{sec}$ intertrial intervals.

The monkeys were deprived of water for $\sim 20 \mathrm{hr}$ before their daily experimental sessions, and the sessions were terminated when a behavioral sign of satiety was observed. At the end of each experimental session, the monkey was returned to its home cage where it was given chow ad libitum. All procedures related to animal care and experimentation were in accordance with the National Institutes of Health Guide for the Care and Use of Laboratory Animals (1996) and were approved by the institutional animal experiment committee.

Procedures of surgery and recording. A stainless steel recording chamber 

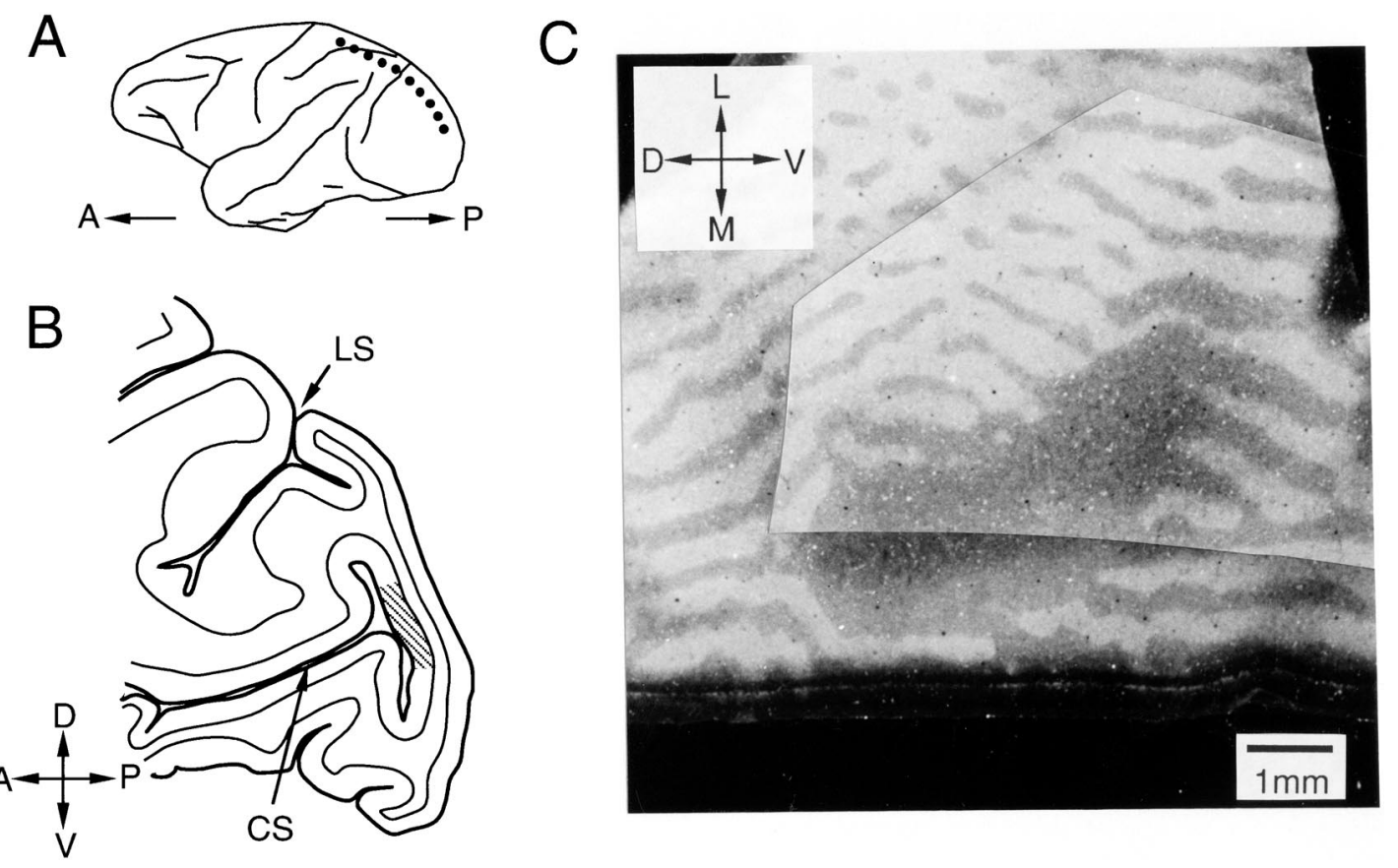

Figure 1. Histological demonstration of the BS representation in V1. $A$, Schematic diagram showing the lateral surface of the macaque cerebral cortex. The dotted line indicates where the section illustrated in $B$ was cut. $B$, Parasagittal section through a posterior region of the cerebral cortex. The hatched region in the posterior wall of the calcarine sulcus $(C S)$ represents an approximate location of the BS representation in V1. $C$, TMB-stained tangential section through the posterior wall of the CS. WGA-HRP was injected into the contralateral eye before killing the animal; the HRP-labeled region appears light, whereas the unlabeled region is dark. $A$, Anterior; $D$, dorsal; $L$, lateral; $L S$, lunate sulcus; $M$, medial; $P$, posterior; $V$, ventral.

and a socket for connecting the monkey's head to a primate chair were fixed to the skull using standard sterile surgical techniques under sodium pentobarbital anesthesia. A search coil was also surgically placed under the conjunctiva of one eye using the method of Judge et al. (1980) and was connected to a plug on the top of the skull. The recording chamber, the socket, and the eye coil plug were all embedded in acrylic that covered the top of the skull and was connected to the skull by implanted bolts.

After surgery, the monkeys were allowed to recover for at least 1 week before the experiments were begun. After surgery but before beginning experimentation, the location of the blind spot of each eye was determined using a monocular visual saccade task, as described previously in detail (Komatsu and Murakami, 1994a). Briefly, each monkey was trained to make a single visual saccade to a small target (a square of $20 \times 20 \mathrm{~min}$ of arc; $20 \mathrm{~cd} / \mathrm{m}^{2}$ ) presented somewhere on the screen (gray background; 1.7 $\mathrm{cd} / \mathrm{m}^{2}$ ) and to maintain fixation otherwise. After the training, the location of the blind spot was determined in a monocular-viewing condition. In most trials, target positions were deliberately chosen to avoid the blind spot, and the monkey was readily able to make a saccade to the target. Intermingled with these trials were others in which the target was presented at a position expected to be near the blind spot, and the ability of the monkey to make a saccade to the target was then used to determine the boundary of the blind spot. The locations and extents of the blind spots proved to be similar in the three monkeys, the blind spots being located approximately along the horizontal meridian at $\sim 16^{\circ}$ from the fixation spot and extending $\sim 5^{\circ}$ horizontally and $7^{\circ}$ vertically (e.g., see Figs. $2,4-7$ ).

Visual responses of neurons were examined using stationary rectangular stimuli $\left(20 \mathrm{~cd} / \mathrm{m}^{2}\right)$ presented on a gray background $\left(1.7 \mathrm{~cd} / \mathrm{m}^{2}\right)$. For the receptive field mapping, square stimuli $0.3-1.3^{\circ}$ on a side were used. To examine the neural responses during the occurrence of perceptual filling-in at the blind spot, we presented rectangular stimuli (referred to as "surface stimuli") that completely covered the blind spot (usually $6.7 \times 10^{\circ}$; occasionally $10 \times 10^{\circ}$ ). Such a stimulus is perceived as a uniformly painted figure even though there is no retinal input at the blind spot; in other words, perceptual filling-in occurs for such a surface stimulus. The fixation spot was presented at a somewhat shifted position, either to the left or right of the screen, so that the entire blind spot was within the screen. To test neural responses under monocular-viewing conditions, one of the eyes was frequently occluded by an opaque mask.

The recording chamber was placed on the occipital cortex and tilted $\sim 55^{\circ}$ posteriorly. A thin-wire elgiloy microelectrode coated with parylene (Microprobe, Potomac, MD) or a glass-coated tungsten microelectrode was advanced in a parasagittal plane using a hydraulic microdrive (Narishige, Tokyo, Japan). Neural signals were amplified and discriminated on the basis of amplitude windows set at two different timings (Bak DDIS-1, Germantown, MD) and then converted to pulses. The unit pulses and behavioral events were recorded on a computer at a temporal resolution of $1 \mathrm{msec}$ and displayed in real time. Eye positions were also monitored every $1 \mathrm{msec}$.
Histology. After completion of the recording experiments, two of the monkeys were deeply anesthetized with sodium pentobarbital and perfused through the heart with fixative; one monkey (BM14) received $0.8 \%$ paraformaldehyde plus $1 \%$ glutaraldehyde, whereas the other (BM23) received $10 \%$ formalin. Each brain was then removed from the skull, and the occipital cortex from the recorded hemisphere was cut into a series of $50 \mu \mathrm{m}$ parasagittal sections. The sections were then stained with cresyl violet to identify the recording sites.

The third monkey is still alive; judging from the penetration site, there is no doubt that recordings were made from V1.

Monkey BM14 was also injected in one eye with wheat germ agglutininconjugated horseradish peroxidase (WGA-HRP; $5 \%$ solution; $20 \mu \mathrm{l}$ ) under sodium pentobarbital anesthesia. The eye was injected twice with a $3 \mathrm{~d}$ interval in between. Five days after the second injection of WGA-HRP, the monkey was perfused. The unrecorded occipital cortex was then sectioned in tangential planes parallel to the cortical surface. Every second section was processed using the tetramethylbenzidine (TMB) method (Mesulam, 1978; Mesulam et al., 1980), whereas the others were stained for cytochrome oxidase. The TMB-stained sections were microscopically inspected under dark-field illumination to detect any trans-synaptically transported HRP label in V1; the BS representation was identified by the absence of label.

\section{RESULTS}

\section{Representation of the blind spot in $\mathbf{V} 1$}

We recorded single-unit activities from three hemispheres of three monkeys. Figure 1 shows the recording sites in V1 where the BS representation is located. This region is shown in Figure $1 B$ as a hatched region in the posterior bank of the calcarine sulcus in a parasagittal section of the occipital lobe (Fig. $1 A$, dotted line). A tangential section through layer 4 of the cortex in the posterior wall of the calcarine sulcus is shown in Figure $1 C$. TMB staining after WGA-HRP injection into one eye clearly shows the alternating labeled and unlabeled stripes identifying the ocular dominance columns, as well as a large unlabeled elliptical region corresponding to the optic disk of the injected eye. Although we will refer to this region as the BS representation, in fact it means "the representation of the region of the visual field corresponding to the blind spot of one of the eyes."

We identified this region by systematic receptive field mapping. Because receptive field positions change in a regular manner along the cortex, we were able to determine precisely the BS represen- 

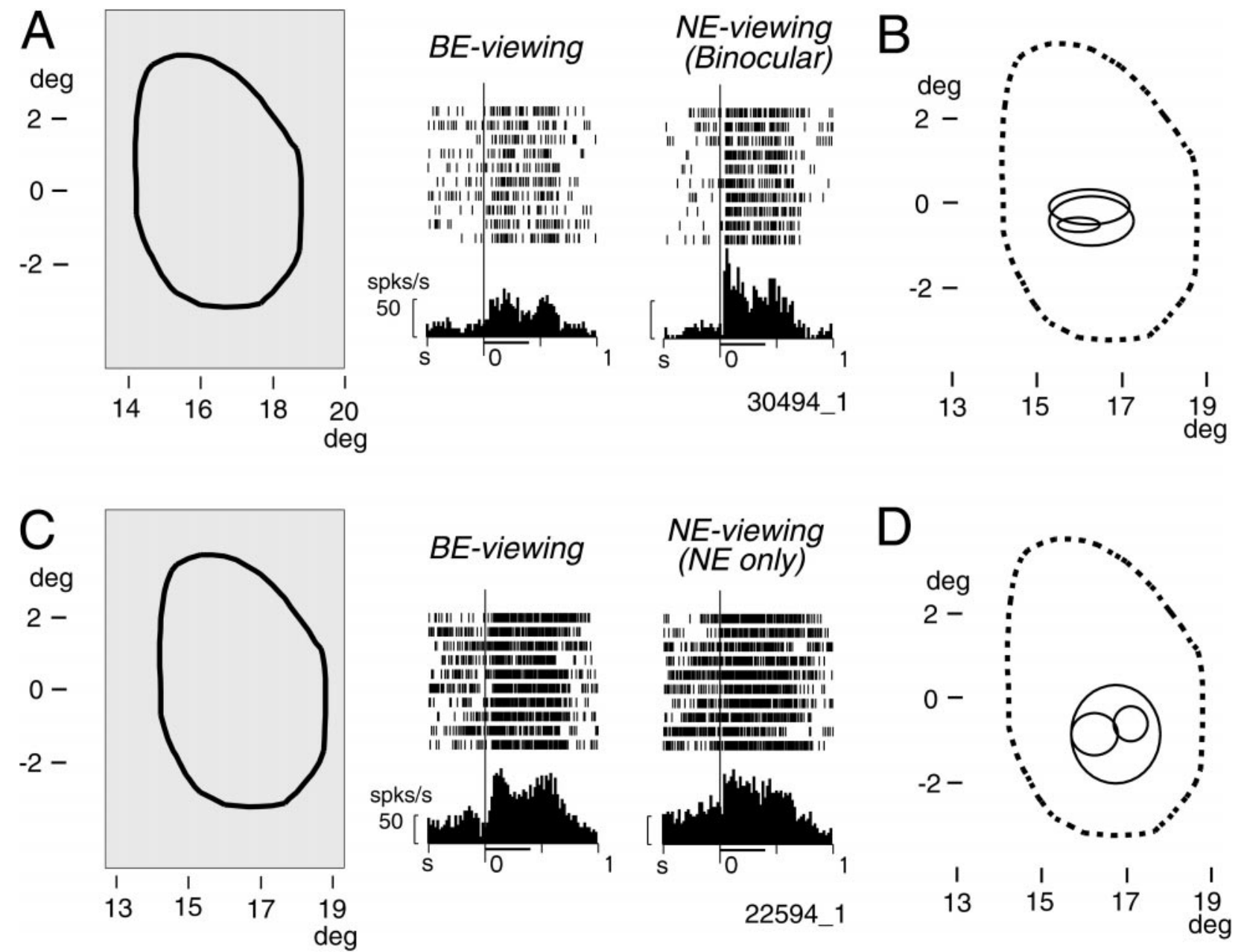

Figure 2. Responses of two representative V1 neurons activated by visual stimuli presented at the blind spot. $A$, Responses of a neuron (30494_ 1 ) to a large homogeneous white rectangular stimulus (surface stimulus) covering the entire blind spot. Left, The spatial relationship between the stimulus, which extended $6.7^{\circ}$ horizontally and $10^{\circ}$ vertically (thin line forming a rectangle), and the blind spot (thick line). Middle, Responses recorded while only the BE was open (BE-viewing). Right, Responses recorded while the NE was open (NE-viewing). PSTHs and rasters are aligned with the onset of the stimuli, which were presented for $400 \mathrm{msec}$ and are indicated by the thick horizontal line below each PSTH. Short vertical lines on the raster display indicate cell discharges; successive lines represent successive trials. In these PSTHs and those in other figures, the tick marks on the $x$-axis are every 500 msec, the bin width is $20 \mathrm{msec}$, and the calibration bar at the left of each histogram is 50 spikes/sec. $B$, Visual field representation of the recording site of the neuron in $A$ as indicated by the receptive fields of other neurons recorded from the same penetration. The boundary of the blind spot is indicated by a broken line. Receptive fields of three single and multiple units (solid lines) recorded in the binocular condition are superimposed. They were located clearly inside of the visual field corresponding to the blind spot. $C$, Responses of another neuron (22594_1) to the surface stimulus. Conventions for rasters and PSTHs are as described in $A$. D, Visual field representation of the recording site of the neuron in $C$ as indicated by the receptive fields of three other neurons recorded from the same penetration. For the sake of simplicity, all receptive fields are plotted as if they were located in the right hemifield in this and the following figures. deg, Degree; $s$, sec; spks, spikes.

tation in V1. When only the eye contralateral to the recorded hemisphere was opened, the blind spot was formed in the examined visual hemifield because the optic disk is located in the nasal hemiretina of each eye. For simplicity, the contralateral eye will be referred to as the blind-spot eye (BE), and the ipsilateral eye will be referred to as the nonblind-spot eye (NE). The viewing condition in which only the BE is open will be called "BE-viewing." When the $\mathrm{NE}$ is open, no blind spot is formed in the examined visual hemifield, and the BS representation in $\mathrm{V} 1$ can receive retinal input. This viewing condition will be called "NE-viewing." NEviewing includes binocular viewing and monocular viewing during which only the NE is open.

\section{Neural responses to surface stimuli}

After the BS representation in V1 was identified, we tested whether neurons in this area responded to surface stimuli covering the blind spot. The rasters and peristimulus time histograms (PSTHs) in Figure 2, $A$ and $C$, show two examples of V1 neurons that clearly responded to the surface stimulus covering the region corresponding to the blind spot. Such responses were observed not only when retinal inputs to this part of the visual field existed (NE-viewing), but also when there was no retinal input to this part of the field (BE-viewing).

The receptive fields of other neurons recorded from the same penetrations are shown in Figure 2, $B$ and $D$. These receptive fields, recorded while NE-viewing, were clearly located inside the region of the visual field corresponding to the blind spot, confirming that our penetrations were indeed made into the BS representation in V1. Figure $2, B$ and $D$, does not include the receptive fields of the neurons in $A$ and $C$, which will be described below.

We examined the responses of 82 single neurons to a surface stimulus presented while BE-viewing (18 from monkey BM14, 60 from BM23, and 4 from GIN). A neuron was regarded as responsive to the surface stimulus if the following two criteria were met: the magnitude of the evoked change in the discharge rate was $>10$ spikes/sec, and the difference between the background discharge rate before the stimulus onset $(0-300 \mathrm{msec})$ and the discharge rate during stimulus presentation $(20-420 \mathrm{msec})$ was statistically significant ( $t$ test, $p<0.05)$. On the basis of these criteria, 20 neurons $(24 \%)$ were classified as responsive to the surface stimulus during BE-viewing (6 from BM14, 12 from BM23, and 2 from GIN). All 


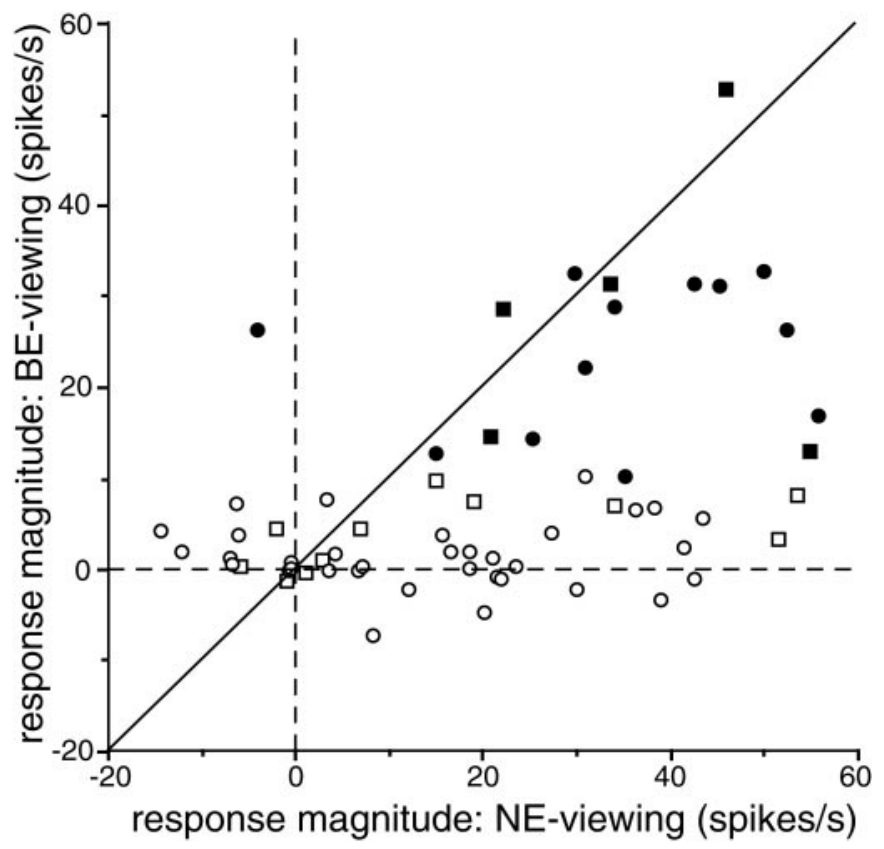

Figure 3. Comparison of responses to surface stimuli obtained under BEand NE-viewing conditions. $y$-axis, Responses obtained while BE-viewing; $x$-axis, those obtained while NE-viewing. Each symbol represents a cell; solid symbols represent cells that responded to the surface stimulus during BE-viewing, whereas open symbols represent those that did not. Squares represent cells whose responses were tested monocularly during NE-viewing; circles represent those tested binocularly during NE-viewing. Broken lines indicate where the response magnitudes are zero. The diagonal line indicates the points at which responses obtained under the different conditions are the same.

of these neurons exhibited excitatory responses. Because the surface stimuli cause perceptual filling-in at the blind spot, the experimental fact so far indicates that some V1 neurons at the BS representation are activated when the animal sees perceptual filling-in.

\section{Properties of neurons responsive to surface stimuli $B E$-viewing versus $N E$-viewing}

The first issue we are going to address is the ocularity of these neurons. The BS representation is basically a monocular region, as evident in the ocular dominance patterns seen in Figure 1. This may imply that in BE-viewing, responses are only a portion of the responses obtained in NE-viewing. To test whether this is the case, we compared the responses to the surface stimulus recorded in $\mathrm{BE}$-viewing and those recorded in NE-viewing in 63 of the 82 neurons recorded (Fig. 3). These include 17 of the 20 classified as responsive during BE-viewing (Fig. 3, solid symbols). Most neurons fell below the diagonal line along which responses obtained during the two viewing conditions were equivalent. This indicates that many neurons were in fact more strongly activated by NE-viewing than by BE-viewing. There were no systematic differences between the results obtained when the NE-viewing was monocular (Fig. 3, square symbols) and those obtained when the NE-viewing was binocular (Fig. 3, circles). Indeed, there were many neurons that responded to NE-viewing but not to BE-viewing; they seem to be monocular cells responding only to stimulation of the NE. Several neurons, however, exhibited comparable responses under both viewing conditions. One responded much more strongly to BEviewing than to NE-viewing. This neuron had an antagonistic off-center/on-surround receptive field organization when mapped during NE-viewing, which resulted in a very weak or even negative response to the surface stimulus. By contrast, a clear excitatory response was evoked during BE-viewing, because the inhibitory center was confined within the blind spot. Therefore, this neuron required a large hole in the middle of the surface stimulus for activation, but other neurons did not.

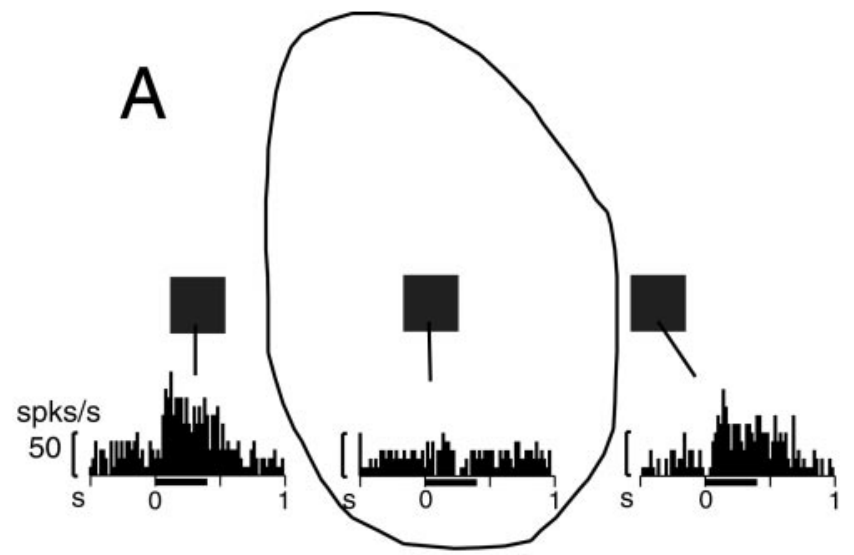

30494

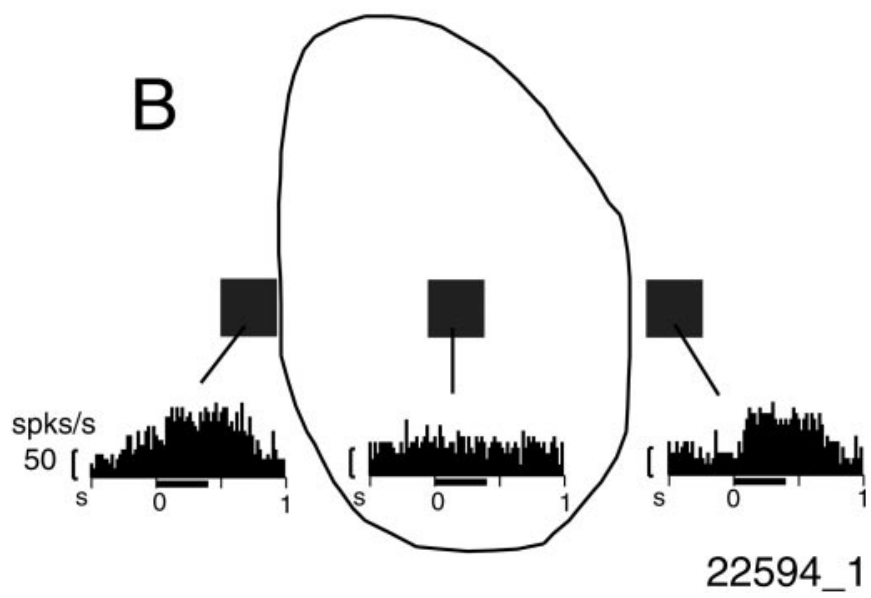

Figure 4. Responses of the same neurons shown in Figure 2, $A$ and $B$, to a small square stimulus $\left(0.67 \times 0.67^{\circ}\right)$ presented inside and outside of the blind spot while BE-viewing. The locations and size of the stimulus relative to the blind spot are shown as solid squares. Responses to each stimulus are shown below the squares as PSTHs.

To summarize, input to this V1 region is predominantly from the $\mathrm{NE}$, but a subpopulation of the neurons also received input from the $\mathrm{BE}$ as well and responded to the surface stimulus during BE-viewing.

\section{Large receptive field}

Because there is no retinal input in the blind spot, neurons responding to a surface stimulus during BE-viewing must have received visual inputs from outside of the blind spot. This may indicate that the receptive fields of these neurons extend out of the blind spot. Alternatively, these neurons may have nonclassical receptive field surrounds around the blind spot and may only be activated by simultaneous stimulation of both sides of the blind spot, as has been suggested by Fiorani et al. (1992). To test these possibilities, we mapped the receptive fields of these neurons using smaller spots $\left(0.33 \times 0.33\right.$ to $\left.1.3 \times 1.3^{\circ}\right)$.

We found that many neurons responding to surface stimuli during BE-viewing also responded when a smaller stimulus was presented outside of the blind spot. For example, the neurons whose responses to surface stimuli are shown in Figure 2 responded vigorously when a small stimulus $\left(0.67 \times 0.67^{\circ}\right)$ was presented on either side of the blind spot (Fig. 4). Another such neuron is illustrated in Figure 5; responses to the surface stimulus are shown in the Figure 5 insets, whereas responses to the small stimulus are plotted at corresponding retinal locations relative to the blind spot. In NE-viewing, the strongest response occurred when the stimulus was presented near the center of the region corresponding to the blind spot (BS-corresponding region). This neuron was also activated by a small spot presented at several 


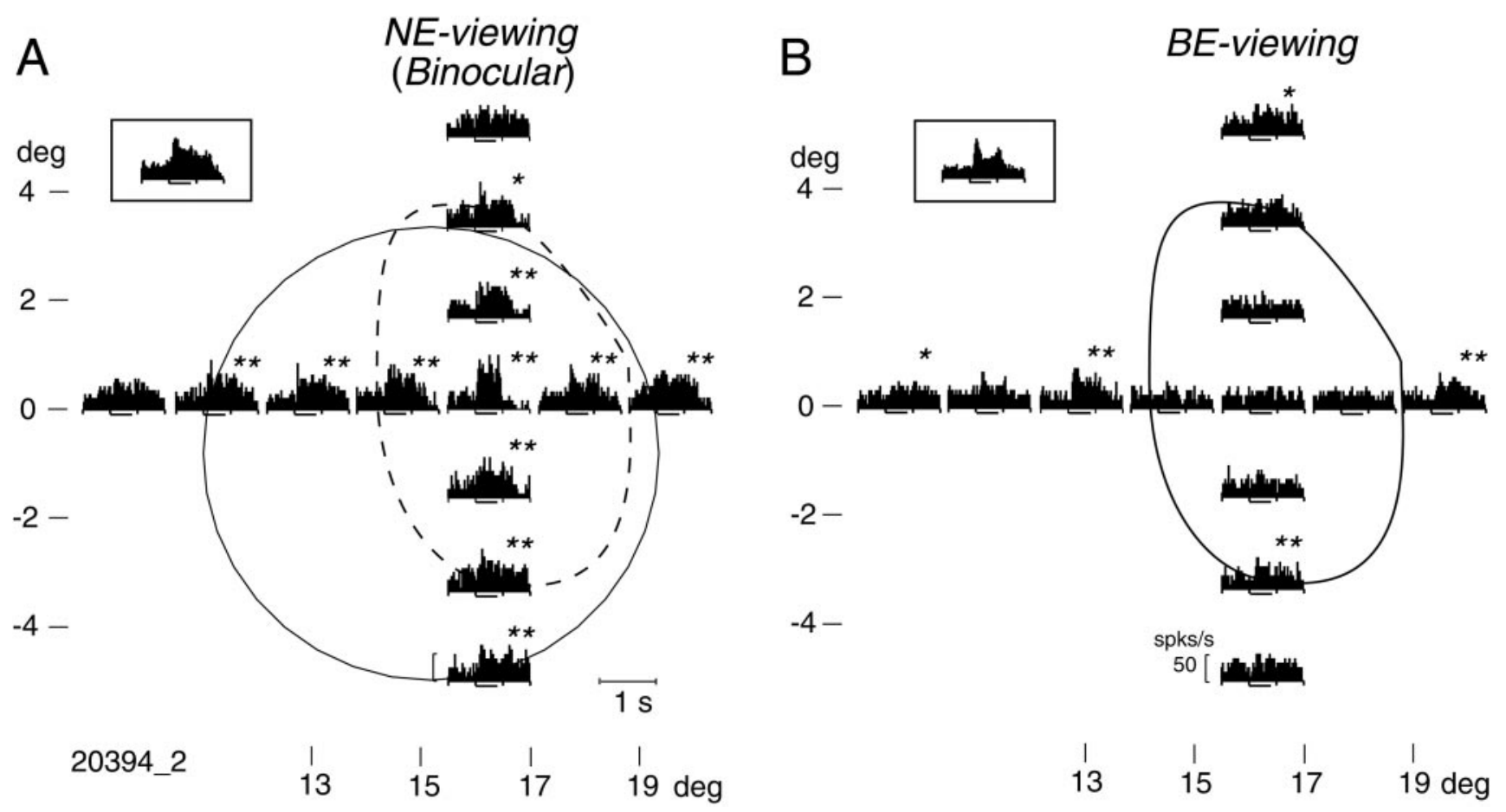

Figure 5. Responses of a neuron to a small stimulus $\left(0.67 \times 0.67^{\circ}\right)$ presented at various locations inside and outside of the blind spot $(B$; solid line $)$ or its corresponding region ( $A$; dashed line). Insets, Responses of this neuron to the surface stimulus. $A$, Responses obtained during NE-viewing. Each PSTH is plotted at the position where the stimulus was presented. Significance of the responses is indicated by asterisks: $* * p<0.01$, and $* 0.01 \leq p<0.05$ in $t$ tests. The boundary of the receptive field was determined as shown by the solid circle. $B$, Responses obtained during monocular BE-viewing. Note that there was no response when the stimulus was presented within the blind spot, but responses to stimuli outside of the blind spot were similar to those shown in $A$.

positions outside of the blind spot during both NE- and BEviewing. In BE-viewing (Fig. $5 B$ ), a small stimulus presented either to the left or right of the blind spot generated clear responses, but there was no response when the stimulus was presented within the blind spot, making it appear as if this neuron had a discontinuous receptive field. The most parsimonious interpretation of this finding is that the neuron had a very large receptive field.

Using a small stimulus, we mapped the receptive fields of the 17 neurons that responded to the surface stimulus. The respective boundaries of the fields were determined by audiomonitoring the responses and by visual inspection of the PSTHs. This was performed while NE-viewing to scan both inside and outside of the BS-corresponding region but was also done in BE-viewing for eight neurons. When responses occurred during BE-viewing, the spatial extents of the receptive fields were generally similar under the two viewing conditions, except that when BE-viewing, cells did not respond to stimuli presented within the blind spot.

The horizontal and vertical extents of the receptive fields of 14 of the neurons are shown in the form of ellipses in Figure $6 \mathrm{~A}$. Of the remaining neurons, two also responded well to stimuli outside of the blind spot, but their data were unsatisfactory for determining the boundary of the receptive field; the other cell failed to respond at all. Thirteen neurons had receptive fields that clearly extended outside the BS-corresponding region. Because we were limited by the edge of the display, in two of the monkeys, the most peripheral visual field mapped was $20^{\circ}$ in eccentricity. Consequently, we may have underestimated the peripheral extent of the receptive field in some of the neurons with very large receptive fields.

These findings indicate that, for many V1 neurons within the BS representation that are activated by stimuli that would yield perceptual filling-in, such activation may be explained by the visual inputs received by receptive fields that are larger than the blind spot. On the other hand, three neurons had receptive fields that were confined to within the BS-corresponding region (Fig. 6A, three plots inside the blind spot). Also, one neuron failed to respond to the small stimulus at any position tested, as described above. Nonetheless, these neurons were activated by the surface stimuli during BE-viewing. Thus, we may need to assume the presence of a nonclassical receptive field surrounding the blind spot for these neurons, as in the "completion neurons" described by Fiorani et al. (1992).

The receptive fields of 51 neurons that did not respond to the surface stimulus during BE-viewing are shown for comparison in Figure $6 B$. In this case, many receptive fields densely covered the inside of the BS-corresponding region; only a few neurons had large receptive fields extending beyond it. It may be, therefore, that a large receptive field is a notable feature of the neurons responding when filling-in perceptually occurs at the blind spot, although this feature is not strictly unique to them.

\section{Stimulus size-response relation}

The large receptive fields of the neurons responsive to the surface stimulus in BE-viewing suggest that these neurons have less position discriminability compared with typical V1 neurons. Then, what about sensitivity to another important spatial parameter, namely, the size of stimuli? To examine the size sensitivity of these neurons, we assessed the relationships between the stimulus size and the response in 10 neurons that responded to the surface stimulus during BE-viewing. A typical series of the stimuli is shown in Figure $7 A$; all were centered either at the center of the cell's receptive field or at the position where the optimum response was obtained within the BS-corresponding region. Presented while NEviewing, the smallest stimulus was much smaller than the blind spot, whereas the largest covered nearly the entire blind spot. Stimulus size-tuning curves of the 10 neurons are shown in Figure $7 B$. The optimum stimulus size differed from cell to cell, but there was a general tendency that the response increased up to $\sim 1-2^{\circ}$ in the stimulus size and then saturated. This tendency can be seen in Figure $7 C$ that indicates the range of the stimulus size that caused the response magnitude greater than half of the maximum for each neuron as solid horizontal bars. In Figure $7 C$, the optimum stimulus size for each neuron is indicated by an open circle. Also, the ranges of stimuli that yielded responses that were not statistically different from the maximum response ( $t$ test, $p>0.05$ ) are indicated by 

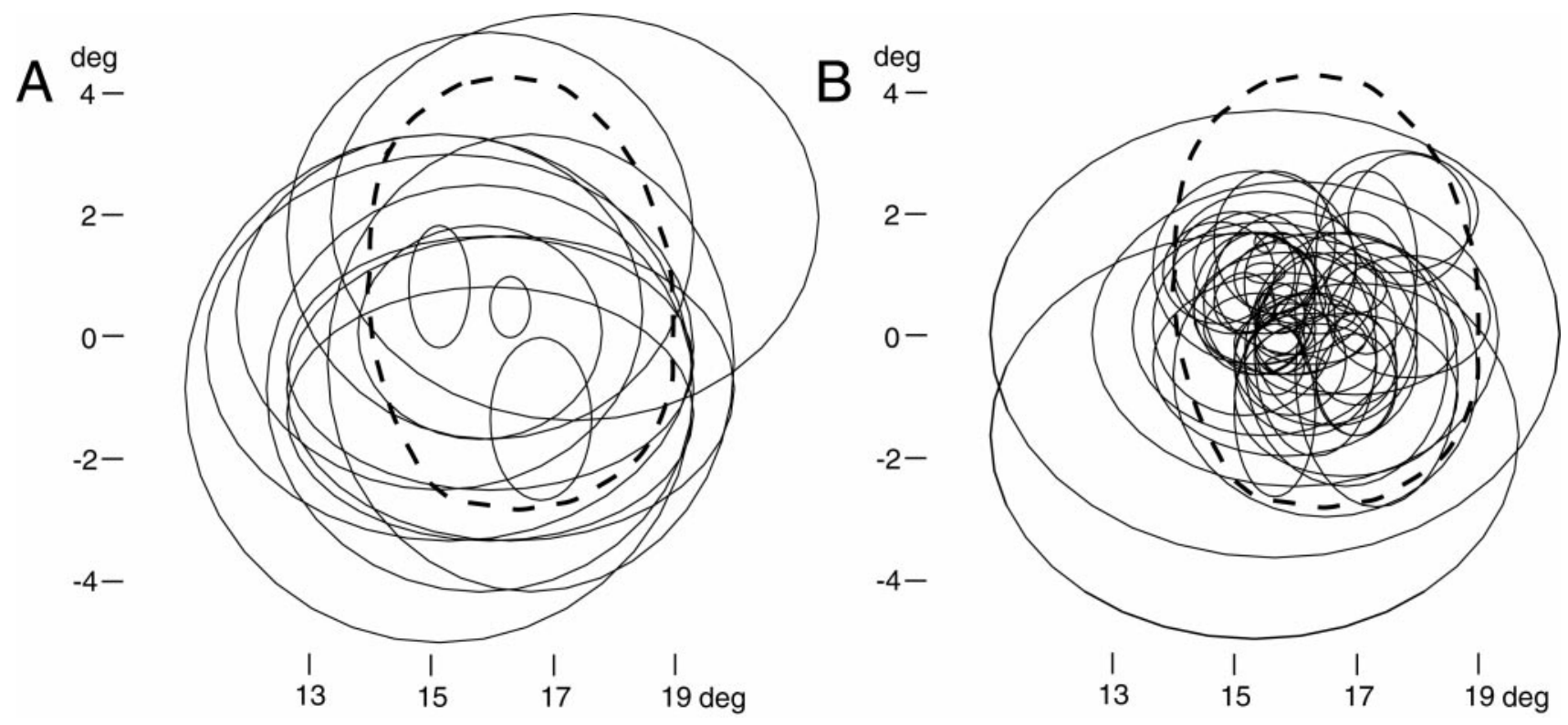

Figure 6. Superimposed plots of the receptive fields of V1 neurons recorded from the BS representation. These receptive fields (thin solid lines) were recorded by the use of small stationary stimuli $\left(0.33 \times 0.33\right.$ to $1.3 \times 1.3^{\circ}$ squares $)$ while NE-viewing. Thick broken lines indicate the boundary of the BS-corresponding region. $A$, Receptive fields of neurons responsive to the surface stimulus. Note that many neurons had large receptive fields that covered most of the blind spot and even extended outside of it. $B$, Receptive fields of neurons that did not respond to the surface stimulus. A few neurons had large receptive fields extending outside of the blind spot.
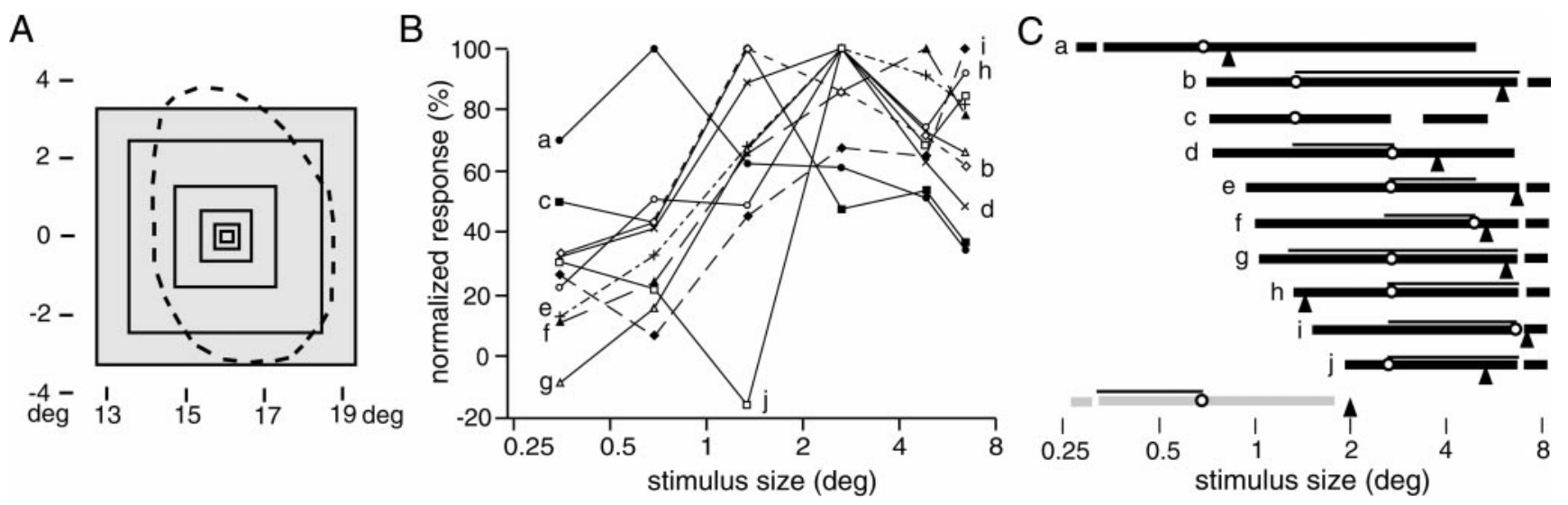

Figure 7. Stimulus size-response relationships of neurons activated by the surface stimulus while BE-viewing. A, Various-sized square stimuli were used to assess spatial summation properties. For each neuron, all stimuli were centered on the same position within the BS-corresponding region (broken line). The actual position differed from cell to cell, however, and a representative example is shown. $B$, The relationship between stimulus size and response magnitude in 10 neurons is shown. The responses were recorded while NE-viewing and are normalized to the maximum response of each neuron. $C$, Ranges of the stimulus size that caused a response magnitude greater than half of that of the optimum size for each of the 10 neurons in $B$ are indicated as solid horizontal bars. Lowercase letters to the left of each bar correspond to those in $B$. The optimum size is indicated by an open circle, and the range of sizes that yielded responses that were statistically not different from the maximum response $(t$ test, $p>0.05)$ are indicated by horizontal lines above each bar. The receptive field sizes $(V$ horizontal extent $\times$ vertical extent) of nine of these neurons are indicated by arrowheads. At the bottom, the range of the stimulus size causing responses in one of the neurons unresponsive to the surface stimulus is indicated as a shaded horizontal bar. A short gap near the left or right ends of the bars indicates that the response was greater than half of the maximum for the smallest or the largest stimuli used.

horizontal lines above each bar. The receptive field size of each neuron is indicated by an arrowhead below each bar. As can be seen, for the stimulus size $>1-2^{\circ}$, the response magnitude was comparable with the maximum response ( $>50 \%$ of the maximum) for most neurons, and in many cases the difference in the response was not statistically significant. Thus it is unlikely that these neurons meaningfully discriminate stimulus size larger than $1-2^{\circ}$. It is also clear that no neuron selectively responded to a smaller stimulus. On the other hand, among those unresponsive to the surface stimulus in BE-viewing, there were many neurons selectively responsive to a smaller stimulus. The range of the stimulus size causing responses in one such neuron is indicated by a shaded horizontal bar at the bottom of Figure $7 C$. These results indicate that neurons that are responsive to surface stimuli carry little information about the precise size of the stimulus, except that they prefer relatively large stimulus sizes compared with typical V1 neurons.

\section{Latency}

When a surface stimulus forms retinal images in two eyes, visual signals are generated by retinal cells throughout the image in the $\mathrm{NE}$, but they are necessarily generated only around the optic disk in the BE. Therefore, when neurons in the BS representation in V1 are activated by the surface stimulus during BE-viewing, visual signals arising from the surround of the blind spot need to be imported into the BS representation. We hypothesized that this might increase the latency of the visual response to the surface stimulus in BE-viewing. The latencies of 15 of the $17 \mathrm{BE}$ responsive cells obtained while $\mathrm{BE}$ - and $\mathrm{NE}$-viewing are plotted in Figure 8 (the other two cells were rejected because they exhibited 


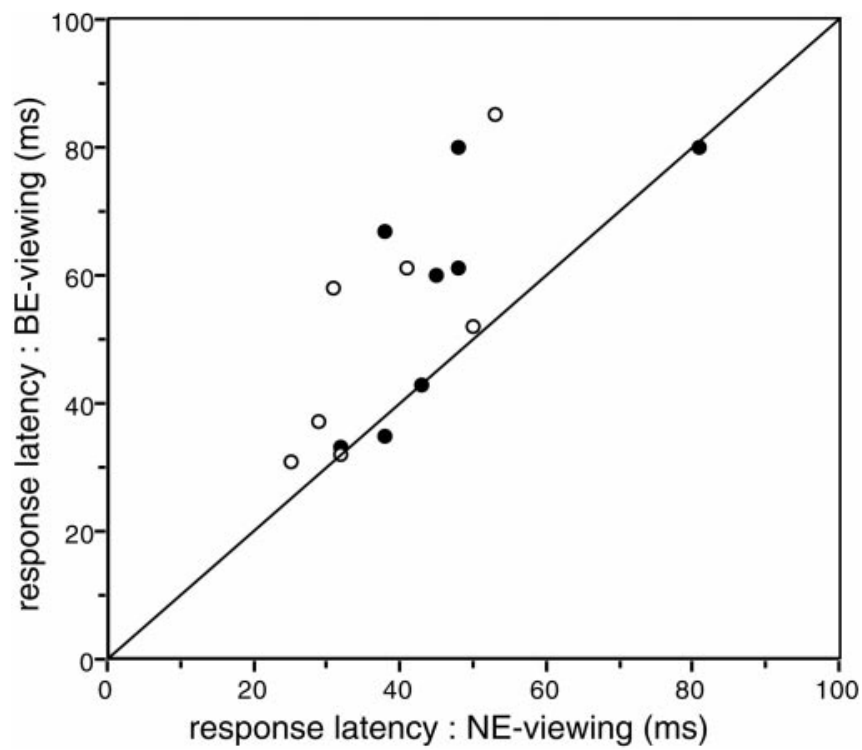

Figure 8. Comparison of response latencies obtained under BE- and $\mathrm{NE}$-viewing conditions. The $x$ - and $y$-axes plot the latencies of responses obtained while NE- or BE-viewing, respectively. For each cell under each condition, PSTHs were convolved with Gaussian $(\sigma=10 \mathrm{msec})$, and the time required for the resultant spike density profile to reach threshold (2 $\mathrm{SD}$ above the background discharge rate) was taken as the latency. Each circle represents a cell; solid circles depict cells in which the magnitudes of evoked responses during a $100 \mathrm{msec}$ period after the latency did not differ significantly ( $t$ test, $p>0.05)$ under the two viewing conditions; open circles depict cells in which response magnitudes did differ significantly under the two viewing conditions. The line indicates where the latency is the same under the two viewing conditions. Note that for most cells, the latency was larger in BE-viewing.

anomalous response fluctuations around the first peak, which made determination of latency unreliable). Differences in the latencies between the two viewing conditions varied from cell to cell, but on average, the latency was significantly ( $p=0.005$, Wilcoxon signed rank test) longer in BE-viewing (mean latency $=54.3 \mathrm{msec}$ ) than in $\mathrm{NE}$-viewing (mean latency $=42.3 \mathrm{msec})$. Figure $9 A$ shows spike density profiles of an exemplar neuron exhibiting latencies of 80 msec during BE-viewing and $48 \mathrm{msec}$ during NE-viewing.

One possible cause of the longer latency associated with BEviewing may be the comparative weakness of the driving force of the visual stimulation, as is suggested by the weaker responses to the surface stimulus (Fig. 3). To test whether this was the case, we computed the mean discharge rate during the initial 100 msec after the response latency for each neuron under both viewing conditions. In Figure 8, the solid circles depict eight cells whose response magnitudes were not statistically different $(t$ test, $p>0.05)$ under the two viewing conditions. Although the difference in the means was not statistically significant ( $p=0.108$, Wilcoxon signed rank test), response latencies clearly tended to be longer in BE-viewing (mean latency $=57.4 \mathrm{msec}$ ) than in NE-viewing (mean latency $=$ $46.6 \mathrm{msec}$ ). The mean response for all eight neurons is shown in Figure $9 B$. Note that the onset of the response occurs at similar latencies under both viewing conditions, but the peak latency is delayed in BE-viewing (94 vs $68 \mathrm{msec}$ ). This divergence in the latencies suggests that there may be more than one pathway by which visual signals are transmitted to the BS representation in V1.

\section{Orientation and color selectivity}

When the perceptual filling-in of a uniform surface occurs at the blind spot, the surface is perceived to be painted with uniform color and brightness. Also, completion of oriented contours occurs at the blind spot. These phenomena suggest that the information about the quality of the surface or contour, such as color, brightness, or orientation, is coded by the neurons involved in the perceptual filling-in at the blind spot. To examine this, we tested orientation selectivity and color selectivity in some of the neurons.
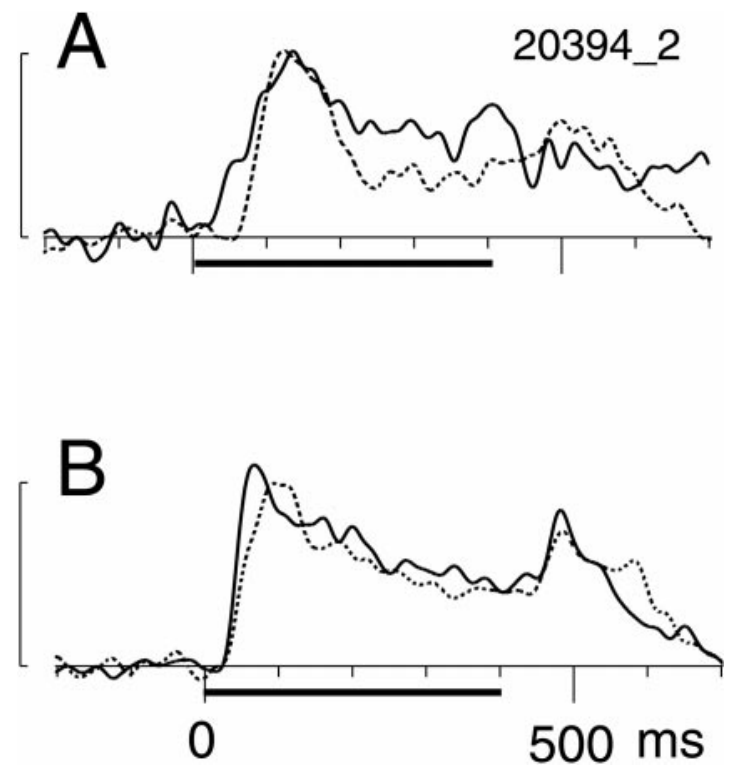

Figure 9. Time courses of the responses to the surface stimulus. Responses are shown as spike density plots in which PSTHs were subjected to Gaussian $(\sigma=10 \mathrm{msec})$ smoothing. In each panel, the dotted line represents $\mathrm{BE}$-viewing; the solid line represents NE-viewing. $A$, Responses of a representative cell in which the magnitudes of evoked responses during a 100 msec period after the latency did not differ significantly ( $t$ test, $p>0.05$ ) under the two viewing conditions. $B$, The mean responses of eight such neurons. The period of stimulus presentation is indicated by the thick horizontal line. The calibrations bars on the left are $50 \mathrm{spikes} / \mathrm{sec}$, and background discharge rates were subtracted from each plot.

$\begin{aligned} & \text { Table 1. Summary of the orientation selectivity and color selectivity of } \\
& \text { neurons }\end{aligned}$
\begin{tabular}{lcr} 
& Orientation selective & Color selective \\
\hline $\begin{array}{l}\text { Cells responsive to the surface } \\
\text { stimulus in BE-viewing }\end{array}$ & $3 / 6(50 \%)$ & $5 / 5(100 \%)$ \\
$\begin{array}{l}\text { Cells unresponsive to the sur- } \\
\text { face stimulus in BE-viewing }\end{array}$ & $16 / 23(69.6 \%)$ & $10 / 16(62.5 \%)$
\end{tabular}

Frequencies of the cells exhibiting selectivity (selectivity index $>0.5 ; p<0.05$, ANOVA) for orientation and color are shown. Frequencies of the cells responsive and those unresponsive to the surface stimulus in BE-viewing are indicated separately.

Orientation selectivity was tested by presenting eight slit stimuli with orientation separated by $22.5^{\circ}$. The stimulus was presented within the receptive field in NE-viewing. Color selectivity was tested by using 9 or 14 colors that were systematically distributed on the Commission Internationale de l'Eclairage-xy chromaticity diagram (Komatsu et al., 1992; Hanazawa et al., 2000). All color stimuli in a given set had the same luminance $\left(4\right.$ or $\left.8 \mathrm{~cd} / \mathrm{m}^{2}\right)$. The color stimuli had a rectangular shape $\left(6.7 \times 10^{\circ}\right.$ in size in most cases) and were presented in NE-viewing in most cases.

Twenty-nine neurons were examined for orientation selectivity; of these, six were among those responsive to the surface stimulus in BE-viewing. For each neuron, we computed the orientation selectivity index defined as follows: 1 - (minimum response)/(maximum response). We classified a cell as selective if both of the following two conditions were satisfied: (1) it had a selectivity index $>0.5$, and (2) responses varied significantly across the set of orientations (one-way ANOVA, $p<0.05$ ). The results are summarized in Table 1. The proportion of orientation-selective neurons was $50 \%$ for those responsive to the surface stimulus, and it was somewhat higher $(69.6 \%)$ for other neurons. Both of these proportions are within the range reported previously for orientationselective neurons in macaque V1 (Felleman and Van Essen, 1987).

Color selectivity was quantitatively determined in the same way that the orientation selectivity was determined. Twenty-one neu- 


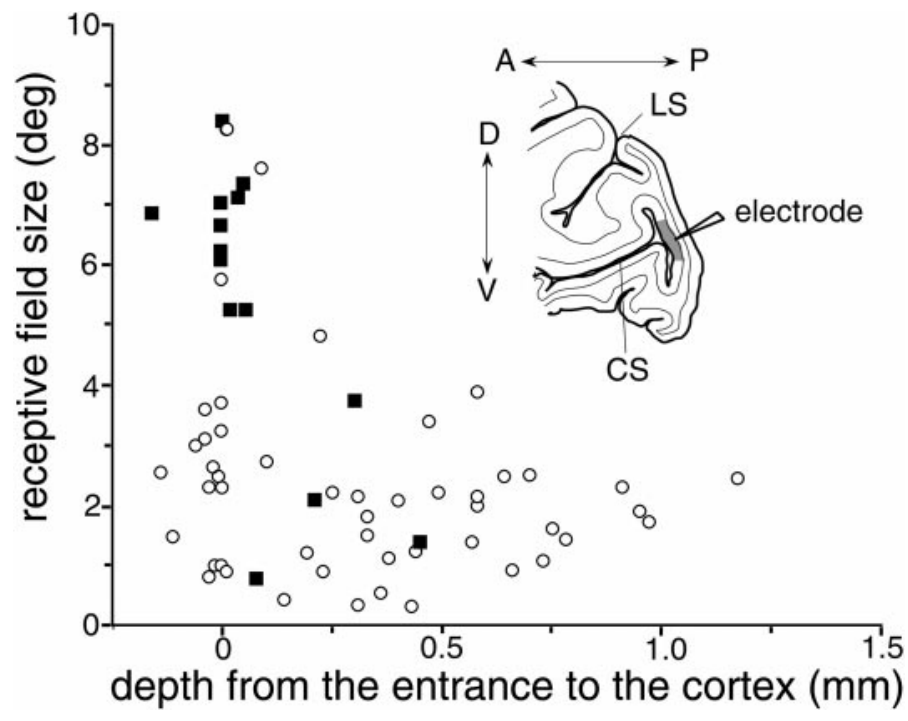

Figure 10. Relationship between recording depth and the receptive field sizes ( $V$ horizontal extent $\times$ vertical extent) of $65 \mathrm{~V} 1$ neurons in the BS representation. Solid squares represent neurons that responded to the surface stimulus while BE-viewing; open circles represent neurons that did not. The $x$-axis shows the distance between the entrance to the posterior bank of the $C S$ and the recording site for each neuron; a value of zero corresponds to layer 6 , whereas a negative value arises when the electrode was retracted slightly to maintain isolation of a single unit. Inset, Schematic diagram showing the electrode track. The electrode first passed through the surface cortex of V1, then passed through the white matter, and finally entered the posterior bank of the $C S$ from layer 6 .

rons were examined for color selectivity; of these, five were among those responsive to the surface stimulus in BE-viewing. As can be seen in Table 1, all five of these neurons were classified as color selective. Of the remaining neurons, 10 were selective.

These results indicate that the activities of neurons that are responsive to the surface stimulus in BE-viewing can convey information about the color of the stimulus and that some of them also convey information about the orientation.

\section{Localization}

Neurons that responded to the surface stimulus covering the blind spot in BE-viewing were not homogeneously distributed in cortical layers. In our experiments, electrodes were advanced into the brain from the posterior surface of the cerebral cortex. As is shown schematically in the Figure 10 inset, the electrode first passed through the posterior surface of V1, then passed through the white matter, and eventually entered the posterior wall of the calcarine sulcus where the BS representation is located. As the electrode approached this region of the cortex, first encountered was layer 6 and then more superficial layers. We realized that neurons responding to the surface stimulus during BE-viewing were often encountered as soon as we entered the cortex at the posterior wall of the calcarine sulcus, most likely at layer 6 .

The relationship between recording depth and the receptive field size of 65 neurons is shown in Figure 10. The depths of the neurons were determined relative to the depth of the first neural activity noticed in the posterior wall of the calcarine sulcus; thus points around zero on the $x$-axis correspond to layer 6 , whereas larger values represent more superficial layers. Negative values on the $x$-axis occurred when the electrode was retracted slightly to maintain isolation of a unit. As was reported previously (Gilbert, 1977), neurons with very large receptive fields were found in layer 6 , and receptive fields rapidly diminished in size as the electrode was advanced. In Figure 10, 14 neurons that responded to the surface stimulus in BE-viewing are represented as solid squares. Ten of them had receptive fields larger than $5^{\circ}$, and they were likely at layer 6. Another neuron was recorded from a depth very close to the point at which the electrode entered this region of cortex, but it had a small receptive field. The remaining three neurons were recorded between 0.2 and $0.5 \mathrm{~mm}$ from the entrance and had receptive fields of more typical size.

Except for those in layer 6, it was difficult to identify the layer of each neuron, because we did not mark each recording site. Nonetheless, receptive field sizes and background discharge rates provided some hints about the layers in which the neurons were situated. The receptive fields were on average the smallest at $\sim 0.3$ $\mathrm{mm}$ from the entrance to the cortex, suggesting that layer $4 \mathrm{C}$ was located there (Fig. 10). In addition, background discharges abruptly dropped at $\sim 0.6 \mathrm{~mm}$ from the entrance to the cortex, suggesting that layer 4 ends at this depth. We thus assume that the three neurons (solid squares) recorded between 0.2 and $0.5 \mathrm{~mm}$ from the entrance to the cortex were sampled from layer 4 or 5 . Among our limited sample, no neuron that responded to the surface stimulus during BE-viewing was found in the superficial layers. This suggests that the neurons within the BS representation of V1 that are activated by stimuli inducing perceptual filling-in are located in layer 4 and deeper, particularly in layer 6 .

\section{DISCUSSION}

The present study provides unequivocal evidence that there are neurons in the BS representation in V1 that are activated when the animal sees perceptual filling-in at the blind spot (Komatsu and Murakami, 1994a). Most of these neurons had very large receptive fields (larger than the blind spot itself), preferred relatively large stimuli, were driven by stimulation of the normal eye as well, were color selective, and were predominantly located in deep cortical layers.

\section{Neural mechanisms of filling-in at the blind spot}

An intriguing question about the neural mechanisms of perceptual filling-in is how the perception of surface attributes is related to the neuronal activity in the retinotopic map of the visual cortex. Theoretically, one extreme possibility is that only the contrast at the edge of a surface area is coded in the central visual system and that neural activity does not correspond topographically to the perception (see Pessoa et al., 1998). This view assumes that filling-in is a result of some higher process. Another view argues that surface-specific neuronal activity in the visual system occurs at a relatively early stage of visual processing and that the activity is topographically correlated with the perceptual consequence of filling-in. Although some psychophysical studies support the former idea (Cumming and Friend, 1980), other studies support the latter (Tripathy and Levi, 1994; Murakami, 1995).

The present study and that of Fiorani et al. (1992) provide physiological evidence that neuron activity occurs at the BS representation when visual stimuli inducing perceptual filling-in are presented across the blind spot. These findings reject the idea that the BS representation in V1 is entirely governed by the normal eye.

In contrast with the experiments done by Fiorani et al. (1992) that used stimuli for completion of the contour, our stimuli induce filling-in of a homogeneous surface. To encode the presence of a uniform surface covering the blind spot, it seems that two sets of information have to be represented. One set of information is the presence of large stimuli with certain color and brightness. The other set of information is the absence of small stimuli, line terminations, local contrast, etc., within this region of the visual field. The activity of cells with little size discriminability but with color selectivity can encode the presence of colored stimuli; thus the activities of the neurons with large receptive fields reported in the present study may carry the first set of information. However, there should be no contradictory information from neurons that are selective for size and position of local contrast. Size-response relations of neurons as shown in Figure 7 suggest that when the surface stimulus covers the blind spot in BE-viewing, cells tuned to smaller sizes were silent to the surface stimulus and thus tacitly carry the second set of information to higher cortical areas. Therefore, both sets of information essential for filling-in seem to be represented in the BS representation in V1. 
Neurons with large receptive fields clearly indicate the presence of some mechanism by which visual information is imported from the surround into the BS representation. Although we can only speculate on what these neurons are actually doing, it is interesting to note that they are mainly localized in layer 6. Neurons in this layer send projections to the extrastriate cortex, but the major target of neurons in layer 6 of V1 is the lateral geniculate nucleus (LGN) (Fitzpatrick et al., 1994). Presumably, therefore, some of the neurons responding during the occurrence of filling-in would send feedback signals to the LGN. It has been suggested that such corticogeniculate feedback modulates visual responses of neurons in the LGN, serving the process of feature binding (Sillito et al., 1994). If layer 6 neurons in the BS representation in V1 send axon collaterals to both sides of the BS representation in the LGN, the feedback signal may modulate neural responses to surface stimuli in the LGN and subsequently in V1. Such a recurrent process might further enhance the activities of V1 and extrastriate neurons responsive to surface stimuli and eventually contribute to perceptual filling-in of a surface across the blind spot.

The above discussion is concerned with perceptual filling-in of simple visual stimuli. Filling-in of more complex patterns also occurs at the blind spot (Kawabata, 1983; Ramachandran, 1992). Undoubtedly, the encoding of complex patterns requires detection of global features across a wide spatial extent and the involvement of higher visual areas.

\section{Comparison with a previous study}

Fiorani et al. (1992) reported previously that some neurons in the BS representation in V1 of the Cebus monkey are activated when a slit stimulus is moved across the blind spot. The present study differs from their report in several important respects. They used anesthetized animals, whereas we used conscious subjects in whom perceptual filling-in was undoubtedly experienced. In addition, the visual stimuli differed. Fiorani et al. used oriented bar stimuli, whereas we used large homogeneous surfaces; the perceptual counterpart is completion of an oriented bar versus filling-in of a surface.

Fiorani et al. (1992) described neurons having "completion properties" that were only activated by stimulation of both sides of the blind spot. We also found neurons with similar properties, but they were rare. In the present study, many of the neurons activated by stimuli that induce filling-in had very large receptive fields that extended out of both the left and right sides of the blind spot. In $\mathrm{NE}$-viewing, the receptive fields consisted of a continuous area, whereas in BE-viewing, the fields naturally became discontinuous across the blind spot. These neurons may thus correspond to those Fiorani et al. described as having discontinuous receptive fields. Unfortunately, Fiorani et al. provide no information comparing the extents of the receptive field during BE- and NE-viewing; they reported only on neural responses occurring while BE-viewing.

To summarize, although there are common findings, the present study and that of Fiorani et al. (1992) emphasize the importance of neurons with different properties in the BS representation in V1. We emphasize that if V1 is involved in surface filling-in at the blind spot, neurons having large receptive fields likely play a key role by importing visual information from the surround to the inside of the retinotopic representation of the blind spot.

\section{Pathways of visual input}

There are at least three possible pathways via which visual inputs can reach the BS representation in V1. First, neurons in the LGN may directly send axons to this region. Neurons surrounding the gap corresponding to the optic disk in the LGN project mainly to the surround of the BS representation in V1, but axons of some of these neurons may diverge and terminate within the BS representation itself. Second, visual inputs may initially reach the surround of the BS representation in V1 and then be transmitted to the inside by way of intracortical horizontal connections. V1 contains extensive horizontal connections (Gilbert and Wiesel, 1983; Rockland and Lund, 1983) that span up to several millimeters, long enough to transmit visual input from the surround to the center of the BS representation. Third, feedback from extrastriate areas may play a role (Bullier et al., 1996; Lamme et al., 1997; Hupe et al., 1998). Transmission via these indirect pathways would require more time than the direct geniculostriate input. The tendency toward longer response latencies associated with BE-viewing seems qualitatively consistent with this possibility. At this moment, however, we have no further evidence favoring any of these three possibilities.

\section{Relation to physiological studies of other perceptual completion phenomena}

Several investigators have sought neural correlates to perceptual filling-in and completion in the visual cortex. Some have found neural activity in V1 to be correlated with perception, such as brightness induction (Rossi et al., 1996) or amodal completion (Sugita, 1999), whereas others have suggested involvement of higher visual areas in the perception, such as illusory contour perception (von der Heydt et al., 1984) or texture filling-in (De Weerd et al., 1995). Also, differences in the time course among various completion phenomena suggest that different sorts of perceptual completion are mediated by different levels of processing in visual cortical areas. For instance, filling-in at the blind spot occurs instantaneously, whereas the filling-in of texture in artificial scotomata requires several seconds to develop (Ramachandran and Gregory, 1991).

Among the various completion phenomena, filling-in at a monocular retinal scotoma may be particularly relevant to the present study. Binocular retinal lesions at the corresponding positions in the two eyes cause reorganization of the retinotopic map in V1 (Kaas et al., 1990; Gilbert and Wiesel, 1992; Chino et al., 1995). In contrast, monocular retinal lesions do not cause such reorganization but leave the affected portion of V1 unresponsive to stimulation of the lesioned eye (Kaas et al., 1990; Murakami et al., 1997), which is very similar to the case of the BS representation in V1. It would be interesting to know whether neural responses similar to those observed in the present study occur in the region representing the monocular scotoma and to determine whether neural responses observed in the present study are specific to the BS representation or are ubiquitous throughout V1.

\section{REFERENCES}

Bullier J, Hupe JM, James A, Girard P (1996) Functional interactions between areas V1 and V2 in the monkey. J Physiol (Paris) 90:217-220.

Chino YM, Smith III EL, Kaas JH, Sasaki Y, Cheng H (1995) Receptivefield properties of deafferentated visual cortical neurons after topographic map reorganization in adult cats. J Neurosci 15:2417-2433.

Cumming G, Friend $\mathrm{H}$ (1980) Perception at the blind spot and tilt aftereffect. Perception 9:233-238.

Daniel PM, Whitteridge D (1961) The representation of the visual field on the cerebral cortex in monkeys. J Physiol (Lond) 159:203-221.

De Weerd P, Gattass R, Desimone R, Ungerleider LG (1995) Responses of cells in monkey visual cortex during perceptual filling-in of an artificial scotoma. Nature 377:731-734.

Felleman DJ, Van Essen DC (1987) Receptive field properties of neurons in area V3 of macaque monkey extrastriate cortex. J Neurophysiol 57:889-920.

Fiorani M, Rosa MGP, Gattas R, Rocha-Miranda CE (1992) Dynamic surrounds of receptive fields in primate striate cortex: a physiological basis for perceptual completion? Proc Natl Acad Sci USA 89:8547-8551.

Fitzpatrick D, Usrey WM, Schofield BR, Einstein G (1994) The sublaminar organization of corticogeniculate neurons in layer 6 of macaque striate cortex. Vis Neurosci 11:307-315.

Gattass R, Gross CG, Sandell JH (1981) Visual topography of V2 in the macaque. J Comp Neurol 201:519-539.

Gattass R, Sousa APB, Gross CG (1988) Visuotopic organization and extent of V3 and V4 of the macaque. J Neurosci 8:1831-1845.

Gerrits HJM, Vendrik AJH (1970) Simultaneous contrast, filling-in process and information processing in man's visual system. Exp Brain Res 11:411-430.

Gilbert CD (1977) Laminar differences in receptive field properties of cells in cat primary visual cortex. J Physiol (Lond) 268:391-421.

Gilbert CD, Wiesel TN (1983) Clustered intrinsic connections in cat visual cortex. J Neurosci 3:1116-1133.

Gilbert CD, Wiesel TN (1992) Receptive field dynamics in adult primary visual cortex. Nature 356:150-152.

Hanazawa A, Komatsu H, Murakami I (2000) Neural selectivity for hue 
and saturation of colour in the primary visual cortex of the monkey. Eur J Neurosci 12:1753-1763.

Hupe JM, James AC, Payne BR, Lomber SG, Girard P, Bullier J (1998) Cortical feedback improves discrimination between figure and background by V1, V2 and V3 neurons. Nature 394:784-787.

Judge SJ, Richmond BJ, Chu FC (1980) Implantation of magnetic search coils for measurement of eye position: an improved method. Vision Res 20:535-538.

Kaas JH, Krubitzer LA, Chino YM, Langston AL, Polley EH, Blair N (1990) Reorganization of retinotopic cortical maps in adult mammals after lesions of the retina. Science 248:229-231.

Kawabata N (1983) Global interactions in perceptual completion at the blind spot. Vision Res 23:275-279.

Komatsu H, Murakami I (1994a) Behavioral evidence of filling-in at the blind spot of the monkey. Vis Neurosci 11:1103-1113.

Komatsu H, Murakami I (1994b) Huge receptive fields of the neurons in the retinotopic map of the macaque V1 representing the blind spot. Neurosci Res [Suppl] 19:S199.

Komatsu H, Ideura Y, Kaji S, Yamane S (1992) Color selectivity of neurons in the inferior temporal cortex of the awake macaque monkey. J Neurosci 12:408-424.

Komatsu H, Murakami I, Kinoshita M (1995) Spatial summation properties of the macaque V1 neurons in the retinotopic representation of the blind spot. Soc Neurosci Abstr 21:1648.

Komatsu H, Murakami I, Kinoshita M (1996) Surface representation in the visual system. Cognit Brain Res 5:97-104.

Lamme VAF (1995) The neurophysiology of figure-ground segregation in primary visual cortex. J Neurosci 15:1605-1615.

Lamme VAF, Zipser K, Spekreijse H (1997) Figure-ground signals in V1 depend on consciousness and feedback from extra-striate areas. Soc Neurosci Abstr 23:1543.

Mesulam MM (1978) Tetramethyl benzidine for horseradish peroxidase neurohistochemistry: a non-carcinogenic blue reaction product with superior sensitivity for visualizing neural afferents and efferents. J Histochem Cytochem 26:106-117.

Mesulam MM, Hegarty E, Barbas H, Carson KA, Gower EC, Knapp AG, Moss MB, Mufson EJ (1980) Additional factors influencing sensitivity in the tetramethyl benzidine method for horseradish peroxidase neurohistochemistry. J Histochem Cytochem 28:1255-1259.

Murakami I (1995) Motion aftereffect after monocular adaptation to filled-in motion at the blind spot. Vision Res 35:1041-1045.

Murakami I, Komatsu H, Kinoshita M (1997) Perceptual filling-in at the scotoma following a monocular retinal lesion in the monkey. Vis Neurosci 14:89-101.

Pessoa L, Thompson E, Noe A (1998) Finding out about filling-in: a guide to perceptual completion for visual science and the philosophy of perception. Behav Brain Sci 21:723-802.

Ramachandran VS (1992) Blind spots. Sci Am 266:86-91.

Ramachandran VS, Gregory RL (1991) Perceptual filling in of artificially induced scotomas in human vision. Nature 350:699-702.

Robinson DA (1963) A method of measuring eye movement using a scleral search coil in a magnetic field. IEEE Trans Biomed Eng 10:137-145.

Rockland KS, Lund JS (1983) Intrinsic laminar lattice connections in primate visual cortex. J Comp Neurol 216:303-318.

Rossi AF, Rittenhouse CD, Paradiso MA (1996) The representation of brightness in primary visual cortex. Science 273:1104-1107.

Sillito AM, Jones HE, Gerstein GL, West DC (1994) Feature-linked synchronization of thalamic relay cell firing induced by feedback from the visual cortex. Nature 369:479-482.

Sugita Y (1999) Grouping of image fragments in primary visual cortex. Nature 401:269-272.

Tripathy SP, Levi DM (1994) Long-range dichoptic interactions in the human visual cortex in the region corresponding to the blind spot. Vision Res 34:1127-1138.

Van Essen DC, Zeki SM (1978) The topographic organization of rhesus monkey prestriate cortex. J Physiol (Lond) 277:193-226.

von der Heydt R, Peterhans E, Baumgartner G (1984) Illusory contours and cortical neuron responses. Science 224:1260-1262.

Walls GL (1954) The filling-in process. Am J Optom Arch Am Acad Optom 31:329-341.

Wurtz RH (1969) Visual receptive fields of striate cortex neurons in awake monkeys. J Neurophysiol 32:727-742.

Zipser K, Lamme VAF, Schiller PH (1996) Contextual modulation in primary visual cortex. J Neurosci 16:7376-7389. 